\title{
Cefozopran Hydrochloride
}

National Cancer Institute

\section{Source}

National Cancer Institute. Cefozopran Hydrochloride. NCI Thesaurus. Code C92168.

The hydrochloride salt form of cefozopran, a semi-synthetic, broad-spectrum, fourthgeneration cephalosporin with antibacterial activity. 